\title{
Tobacco Mosaic Virus in Tissues of Nicotiana tabacum cv. "Little Turkish"
}

\author{
Egbert W. Henry \\ Department of Biological Sciences, Oakland University \\ Rochester, Michigan, 48063 U.S.A.
}

Received November 18, 1978

The tobacco mosaic virus (TMV) is an example of a plant virus that demonstrates no selective relation to a precise tissue of the host plant (Esau and Cronshaw 1967). There have been earlier reports of the ubiquitous occurrence of TMV inclusions in infected host plants (Esau 1941, Goldstein 1926). Several investigators have reported on the effects of tobacco mosaic virus (TMV) in leaves of Nicotiana tabacum (Weststeijm 1976, Esau and Cronshaw 1967, Edwardson 1966, Weintraub and Ragetli 1964, Favali et al. 1974, Betto et al. 1972, Gerola et al. 1969, Conti et al. 1974, Brants 1964, Kontaxis and Schlegel 1962, Wu 1973, Israel and Ross 1967, Shalla 1968, Zaitlin and Boardman 1958, Cech 1967, Milne 1966).

Ectodesmata may be involved in the initiation of TMV infection (Brants 1964). Basal septa have been suggested as TMV infection sites (Kontaxis and Schlegel 1962), indicating that plasmodesmata in the basal septum of a hair is implicated in viral infection. Esau (1933) reported that TMV caused abnormalities commonly associated with the necrosis of plant vascular systems; however, she cautions that vein banding, vein clearing, lignification and suberization may well be common responses to injury, and not necessarily specific reactions to viruses.

It is generally assumed that there is a relationship between host resistance and changes in phenolic compounds and enzymes of the infected tissue (Goodman et al. 1967). Oxidative metabolism, accompanying TMV infection, can cause an increase in host phenylalanine ammonia-lyase (Conti et al. 1974), polyphenol oxidase and peroxidase (Weststeijn 1976) with a decrease in o-dihydric phenols, especially chlorogenic acid (Farkas et al. 1960). With respect to fine structural alterations within virus infected host tissue, Betto et al. (1972) suggest that the chloroplast may well be a possible site for viral multiplication. It was decided to investigate the ultrastructural appearance of leaf and root cellular organelles in tobacco plants systemically infected with TMV.

\section{Materials and methods}

Twelve-week-old Nicotiana tabacum L. cv. "Little Turkish" plants were grown under normal greenhouse conditions with a temperature of $23 \pm 2^{\circ} \mathrm{C}$ during the day and $20 \pm 2^{\circ} \mathrm{C}$ at night. The plant leaves of experimental plants were lightly dusted with Carborundum powder (600-mesh) and subsequently inoculated with a purified solution of TM4 (Gerola et al. 1969).

Portions of leaf and root tissue were subsequently excised from treated ( 1 week 
after inoculation) and control plants and fixed immediately in $3 \%$ glutaraldehyde $\left(50 \%\right.$ aqueous solution, Fisher) in $0.1 \mathrm{M}$ phosphate buffer, $\mathrm{pH} 7.2$, for $1 \mathrm{~h}$ at $4^{\circ} \mathrm{C}$. The tissue sections were rinsed several times in $0.1 \mathrm{M}$ phosphate buffer, $\mathrm{pH}$ 7.2. Tissue sections were post-fixed with $2 \%$ osmium tetroxide in $0.01 \mathrm{M}$ phosphate buffer, $\mathrm{pH} 7.2$ for $1 \mathrm{~h}$. Dehydration was carried out in a graded ethanol series followed by treatment with propylene oxide, with embedding in Epon 812 (Luft 1961).

Tissue sections were cut on a Porter-Blum ultramicrotome, using glass knives and collected on 300-mesh copper grids. Sections were examined with post-staining uranyl acetate. The prepared sections were examined with a Philips 200 electron microscope.

\section{Results}

The following ultrastructural alterations were observed in organelles of TMV infected leaf and root tissue:

Mesophyle leaf tissue:

1. Chloroplasts: The chloroplasts are swollen and distorted in shape (Figs. $1,5,6,7,10,11-15$ ). The lamellae are swollen (Figs. 5, 6, 13) with outer membrane distortion (Fig. 5).

2. Mitochondria: The mitochondria appear to be swollen (Figs. 1, 3-6, 12) and the cristae show signs of dissolution (Fig. 6). A dense crystalloid core (CC) is present in one mitochondrion (Fig. 12).

3. Ribosomes: Extensive ribosomal activity is present in some meophyll cells (Figs. 3-4, 12).

4. Opaque bodies: Opaque bodies with light perimeter areas occur in some meophyll cells (Figs. 1-2, 6, 10-11).

Root tissue:

1. Mitochondria: The mitochondria are swollen and contain an electron dense body (DB) (Fig. 8).

2. Ribosomes: Extensive ribosomal activity is present in some TMV infected root tissue (Fig. 8).

3. Cell wall: The middle lamellar (M) area of the cell wall is undergoing dissolution (Fig. 8).

4. Nucleus: $A$ "doughnut"-shaped nucleus is present in this root infected tissue (Fig. 9).

\section{Discussion}

TMV appears to move into all portions of the infected leaf and root tissues (Figs. 1-16). The chloroplasts have partially dissolved envelope membranes and are generally distorted in shape, with large starch bodies (Figs. 1, 5-7, 10-15). Some infected chloroplasts have large opaque bodies within the stroma (Figs. 1, 5). The chloroplast envelope appears to become vesiculated near the areas where virus particles become attached to the surface (Figs. 1, 5-7, 10-14). While chloroplasts 

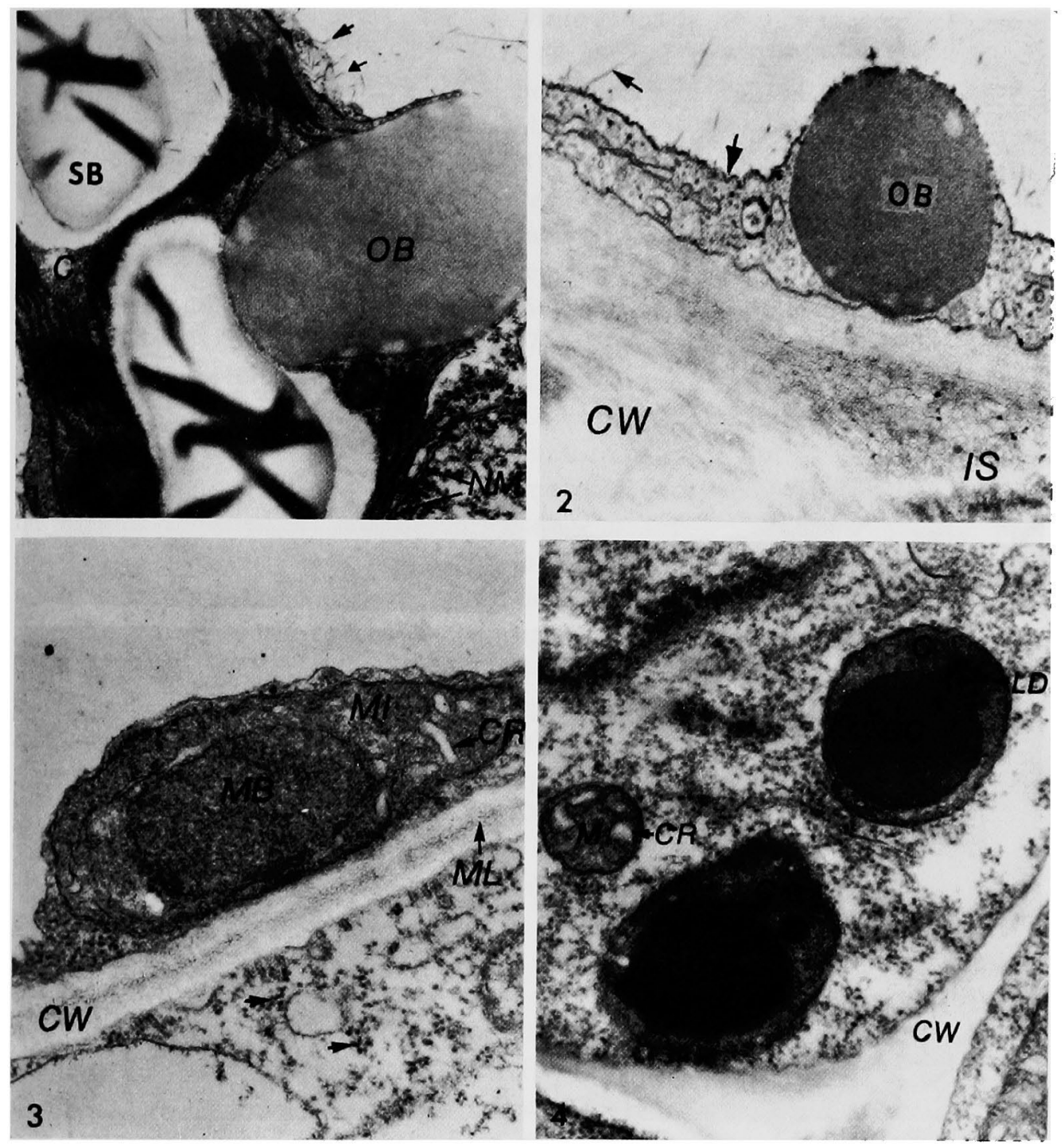

Figs. 1-4. 1, portion of mesophyll cell chloroplast (C) containing a bulging out, slightly opaque, single membrane-bounded body (OB). TMV particles are visible around the perimeters of the outer chloroplast membranes (arrows). Some TMV particles are close to a starch body (SB). Chloroplast lamellae (L) are distorted and the chloroplast contents are swollen. The nuclear membrane (NM) has lost integrity and has become discontinuous (arrows) around the nucleus (N). Osmium tetroxide fixation with uranyl acetate post-staining. $\times 86,000$. 2 , portion of mesophyll cell showing opaque, single membrane-bound body (OB) bulging out from the cytoplasmic area. TMV particles (arrows) are near the outer plasma membrane (PM). The outer plasma membrane (PM) is more electron dense than is the PM bordering the cell wall (CW). Intercellular spaces (IS) of cell wall is under-going dissolution. Osmium tetroxide fixation with uranyl acetate post-staining. $\times 72,000$. 3, portion of mesophyll cell showing a swollen mitochondrion (M) of a TMV infected cell. The cristae (CR) are displaced and a granular, single membrane-bound body (MB) is present within the mitochondrion (M). Extensive ribosomal activity is present in the cellular cytoplasm (arrows). The middle lamellar (ML) area of the cell wall (CW) is beginning to lose its integrity and undergo dissolution. Osmium tetroxide fixation with uranyl acetate post-staining. $\times 57,500$. 4 , portions of three adjacent mesophyll cells of TMV infected leaf. Single membrane-bound granu- 

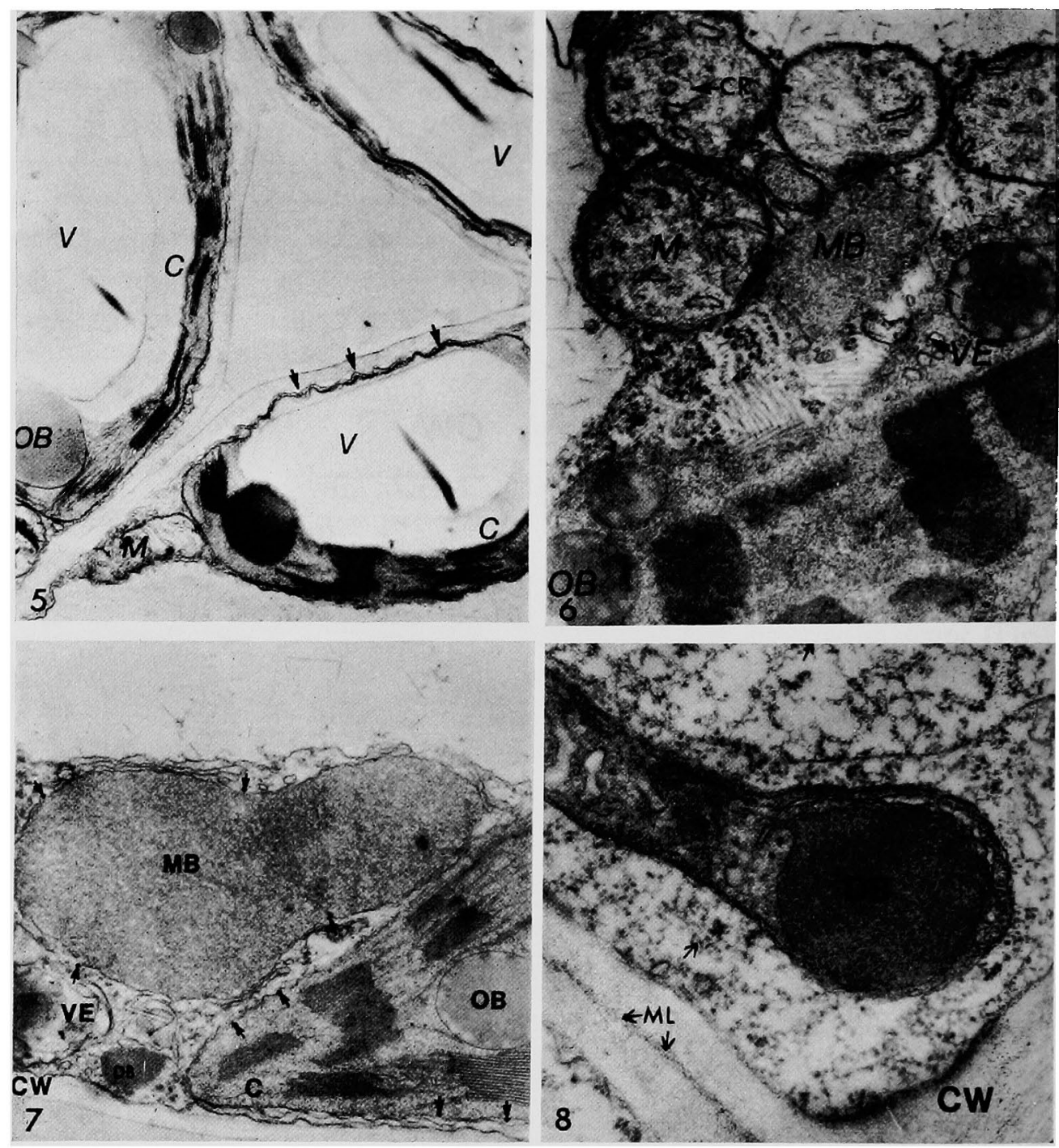

Figs. 5-8. 5, portions of three adjoining mesophyll cells of a TMV infected leaf. The chloroplasts (C) are swollen and disorted in shape. The chloroplast lamellae (L) are swollen. Opaque, single membrane-bound bodies (OB) are present within the cytoplasm of the chloroplast (C). The mitochondria (M) are beginning to show discontinuity along the outer membranes and electron dense (arrows), thread-like deposits are present inside of the mitochondria (M). TMV particles are present in the vacuole (V). The outer chloroplast membranes show discontinuity and interdigitation near the cell wall outer areas (arrows). Osmium tetroxide fixation with uranyl acetate post-staining. $\times 37,500$. 6 , TMV particles (arrows) have invaded the cytoplasm of this mesophyll cell and the mitochondria (M) are showing dissolution within the cristae (CR). The granular portion of a microbody (MB) remains with little evidence of its outer single membrane. The outer chloroplast (C) membranes are not present where the TMV particles are congregated near vesicles

lar components (GC) within two chloroplasts (C) are beginning to swell. Several lipid droplets (LD) are present in one chloroplast (C). A mitochondrion (M) shows signs of swelling, with enlarged cristae (CR). Ribosomal activity is evident throughout the cytoplasm (arrows). The cell wall $(\mathrm{CW})$ has started to separate from the adjacent plasma membrane of one cell (arrows). Osmium tetroxide fixation with uranyl acetate post-staining. $\times 47,500$ 
may be considered a site for possible virus multiplication, systemically infected leaves rarely seem to have virus particles (Betto et al. 1972).

Zaitlin and Boardman (1958) and Boardman and Zaitlin (1958) reported the isolation of TMV diseased-leaf chloroplast fraction homogenates; however, no virus particles were seen by Reddi (1964) in TMV infected tobacco chloroplasts. Ultrastructural studies by Kolehamainen et al. (1965) did not reveal TMV particles in chloroplasts; however, Milne (1966) and Shalla (1964) suggested that chloroplast virus particles were actually representative of chloroplast invaginations that were juxtaposed with the cytoplasm. TMV synthesis in the chloroplast is still an open question (Esau and Cronshaw 1967). Starch lesions were first observed in chloroplasts by Holmes (1929) and it has been suggested that these lesions develop from starch grains that gradually enlarge (Weintraub and Ragetli 1964).

The presence of swollen cristae and mitochondria in both leaf mesophyll and root infected tissue (Figs. 1, 3-6, 8, 12) may be indicative of the TMV-caused enhancement of oxidative metabolism (Farkas et al. 1960). As TMV infection proliferates the respiration of leaf cells attain a level below that of non-infected cells, along with a concomitantly low succinoxidase activity (Weintraub et al. 1964).

The general occurrence of large single membrane-bound microbodies (Figs. $3,6-7,12$ ), opaque bodies (Figs. 1-2, 6, 10-11) and dense bodies (Figs. 7-8), suggests that TMV does alter the metabolism of phenolic compounds (Fritig et al. 1972); moreover, peroxidase, catalase and polyphenol oxidase have been localized via 33-diaminobenzidine (DAB) in similar organelles in tobacco flower pedicel tissue (Henry et al. 1971, Henry and Jensen, 1973, Henry 1974, Henry et al. 1974, Henry 1975a, b).

The general dissolution and degradation accompanying TMV infection in the cell walls (Figs. 3-4, 8), vacuoles (Figs. 5, 13, 15), plasma membrane (Fig. 11), and interstitial spaces (Fig. 2) appears to be a common response of both leaf mesophyll and root tissues to TMV infection.

The large opaque bodies that protrude through the chloroplast envelope membranes are surrounded by virus particles (Figs. 1, 5-7, 10-15) and appear to be unique in appearance in TMV infected tissues.

Doughnut-shaped nuclei have been reported in rat ova during cleavage (Dvorak 1974), in tomato flower pedicel abscission zone tissue (Jensen and Valdovinos

(VE) within the plastid. Some of the chloroplast lamellae (L) are markedly swollen (arrows). Opaque bodies $(\mathrm{OB})$, with several light areas near the perimeter are present within the chloroplast stroma. Osmium tetroxide fixation with uranyl acetate post-staining. $\times 66,000.7$, portion of a mesophyll cell infected with TMV. TMV particles have invaded the cytoplasm of the cell (arrows) and are close to the single membrane-bound microbody (MB) and chloroplast (C). Vesiculation (VE) and loss of microbody membrane integrity is evident (arrows). On opaque, single membrane-bound body $(\mathrm{OB})$ is present in the chloroplast stroma. The outer chloroplast membranes are becoming discontinuous (arrows), particularly near the cell wall (CW). An electron dense body (DB) is becoming surrounded by vesiculation within the cytoplasm. Osmium tetroxide fixation with uranyl acetate post-staining. $\times 63,000.8$, root cell infected with TMV showing a swollen mitochondrion (M) with a single membrane-bound electron dense body (DB) at one end. There is evidence of active ribosomal activity within the cytoplasm (arrows). The middle lamellar (ML) of the cell wall $(\mathrm{CW})$ is beginning to show signs of degradation and dissolution (arrows). Osmium tetroxide fixation with uranyl acetate post-staining. $\times 70,000$ 

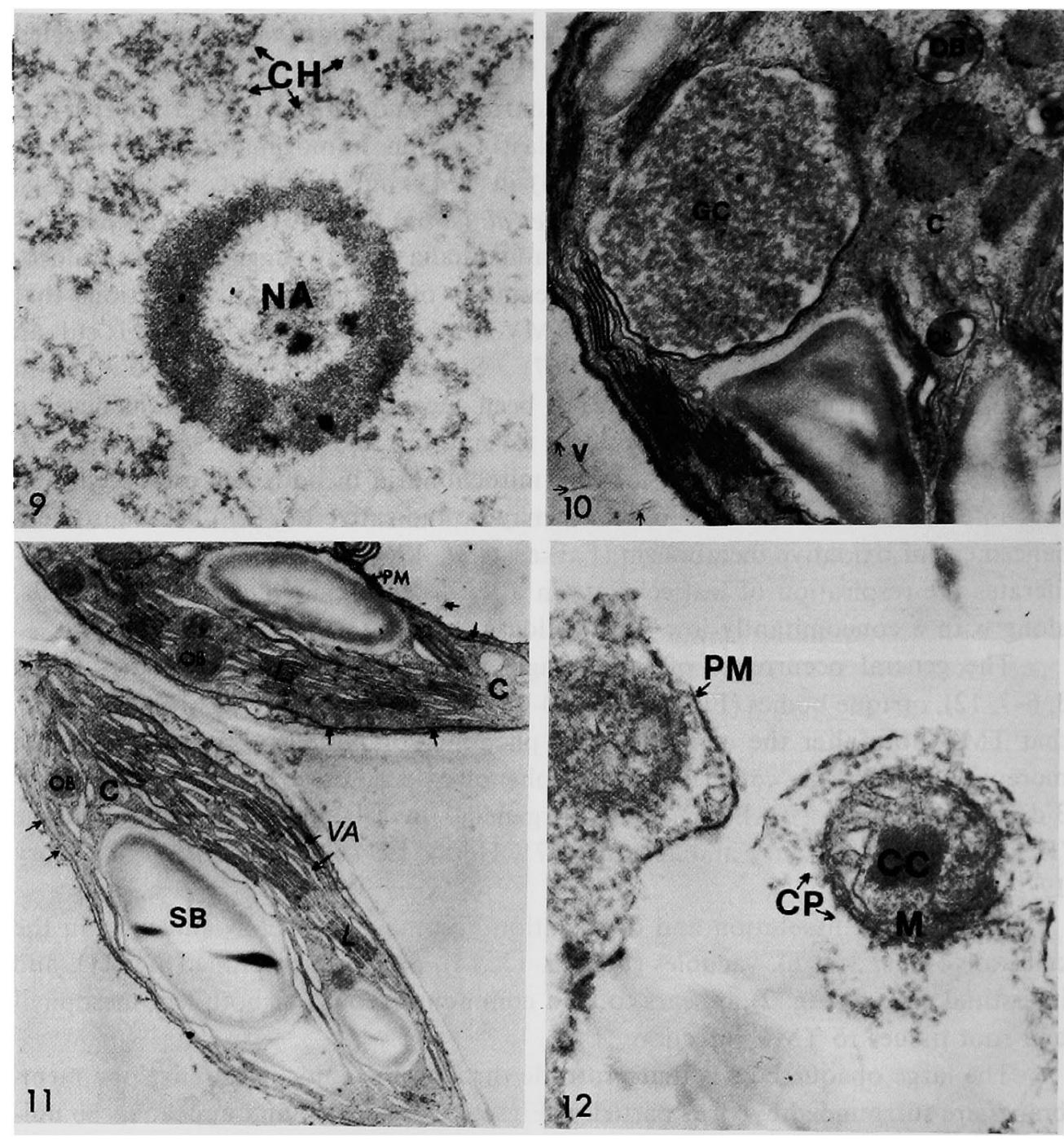

Figs. 9-12. 9, portion of a TMV infected root cell showing a "doughnut"-shaped nucleolus (NA) within the nucleoplasm. Chromatin $(\mathrm{CH})$ is granular and randomly dispersed within the nucleoplasm. Osmium tetroxide fixation with uranyl acetate post-staining. $\times 77,000.10$, chloroplast (C) of TMV infected mesophyll adjacent to vacuole (V) containing TMV particles (arrows). The outer chloroplast membranes are discontinuous and the lamellae (L) are swollen and distorted. A single membrane-bound granular component (GC) is present within the plastid stroma. Several smaller, opaque bodies $(\mathrm{OB})$ with light areas are present in the chloroplast stroma. Three starch bodies (SB) are visible. Osmium tetroxide fixation with uranyl acetate post-staining. $\times 91,000$. 11, two mesophyll cell chloroplasts (C) in TMV infected leaf tissue. The lamellae (L) are swollen (arrows). Light, vacuolate areas (VA) are present in one plastid. Starch bodies (SB) and opaque bodies, (OB) are also present within the plastids. TMV particles are present near the plasma membrane (PM) (arrows). Osmium tetroxide fixation with uranyl acetate post-staining. $\times 43,000.12$, portion of mesophyll of TMV infected leaf showing a mitochondrion (M) with a dense crystalloidtype core $(\mathrm{CC})$. The adjacent cytoplasm (CP) is becoming vacuolate. The dark, plasma membrane (PM) is discontinuous (arrows). There is evidence of ribosomal activity near a mitochondrion (M) undergoing degradation. Osmium tetroxide fixation with uranyl acetate post-staining. 

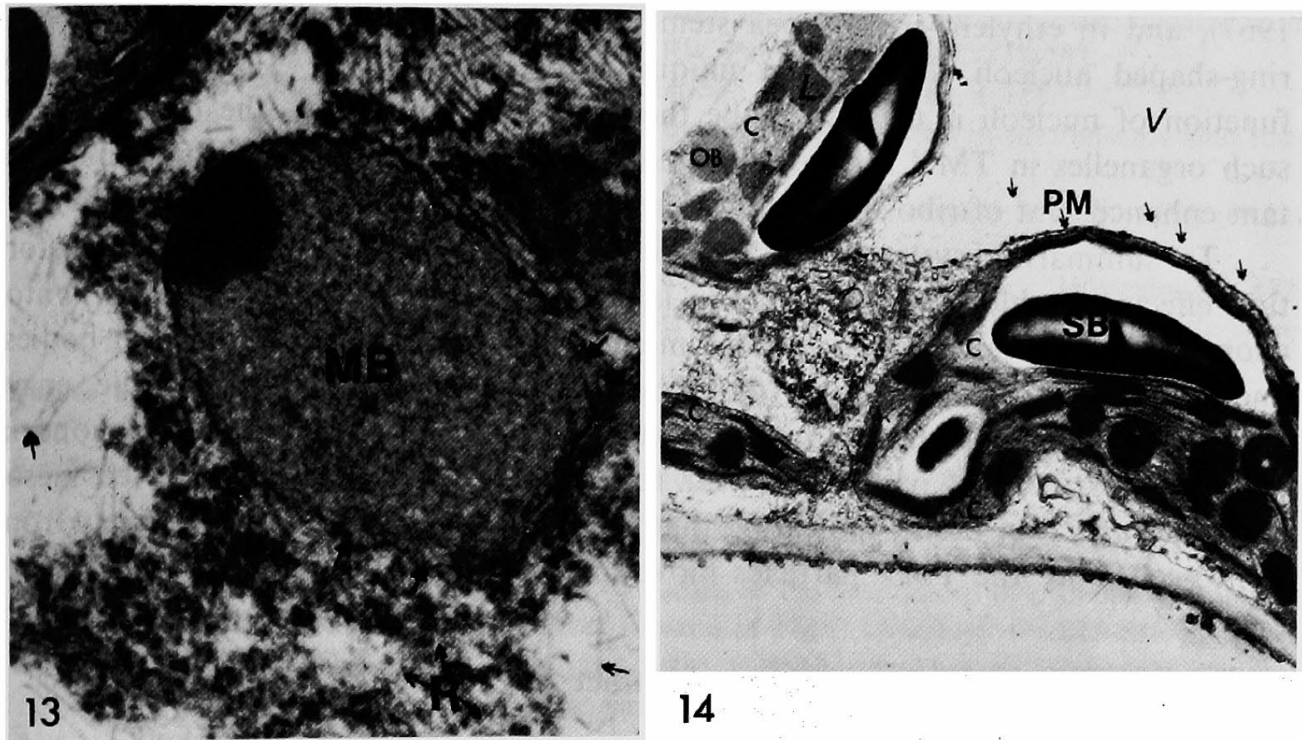

14

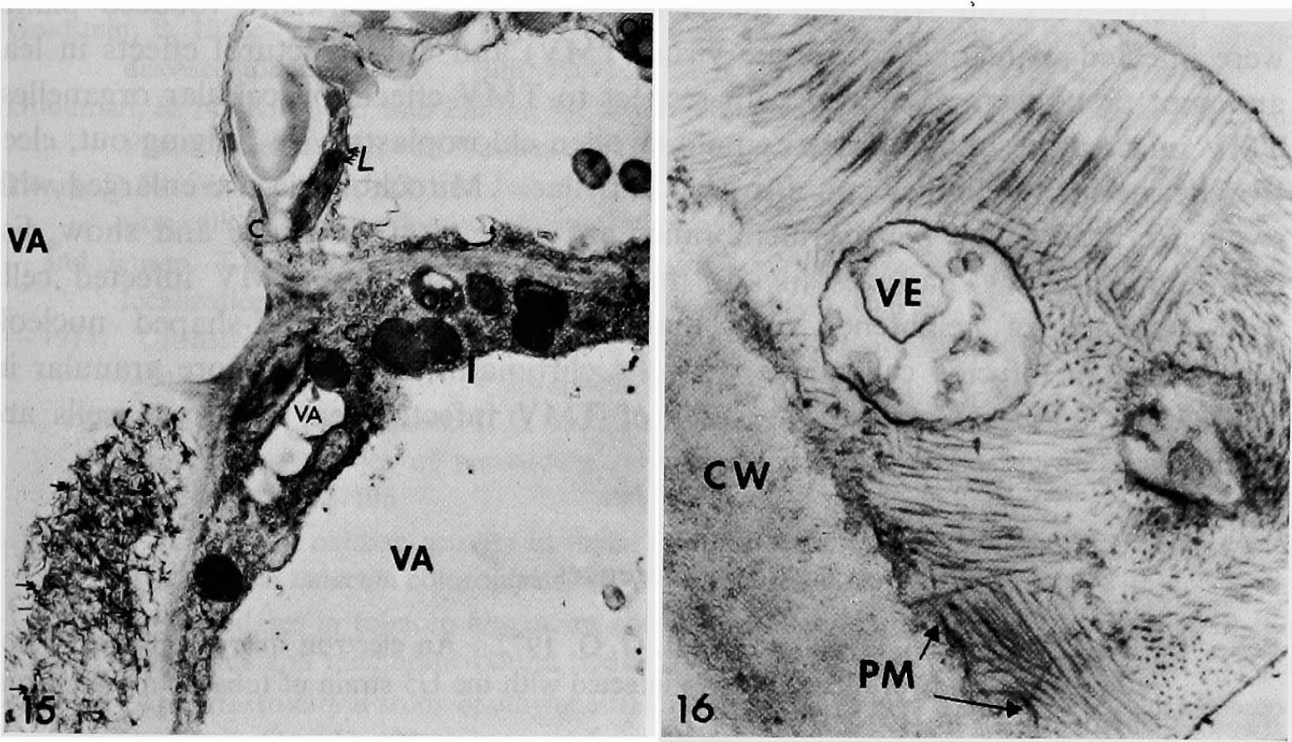

Figs. 13-16. 13, portion of mesophyll cell showing TMV particles (arrows) present in the cytoplasm and surrounding a single membrane-bound microbody (MB). The membrane of the microbody (MB) is becoming discontinuous (arrows) and a crystalloid is present at one end of the microbody (MB). A portion of the chloroplast (C) is present with swollen thylakoids (T). Ribosomal (R) configurations are also present in the cellular cytoplasm. Osmium tetroxide fixation with uranyl acetate post-staining. $\times 57,000$. 14 , a view of TMV infected mesophyll cell chloroplasts (C), showing the proliferation of opaque bodies (OB) within the chloroplast stroma. The chloroplasts are distorted, with swollen lamellae (L). TMV particles (arrows) are visible on the inner edges of the vacuole (V) near the electron dense areas of the plasma membrane (PM). Oversized starch bodies (SB) are present in several of the chloroplasts (C). Osmium tetroxide fixation with uranyl acetate post-staining. $\times 46,500 . \quad 15$, a portion of TMV infected leaf tissue showing several mesophyll cells. Electron dense, opaque bodies (OB) are present in the cellular cytoplasm as well as within the chloroplast (C) stroma. Dense ribosomal areas are present within the cytoplasm. Needle-like congregations of TMV pervade the inner area of one former cytoplasmic area (arrows). There are three adjacent vaculate (VA) areas within the cytoplasm and chloroplast (C) stroma. 
1967), and in ethylene-treated pea stem sections (Henry 1978). The presence of ring-shaped nucleoli is somewhat unique in occurrence (Fig. 9). As a primary function of nucleoli is thought to be the production of rRNA, the occurrence of such organelles in TMV infected host tissue may be correlated with the concomitant enhancement of ribosomal activity.

To summarize, systemic TMV infection appears to have appreciable distortion effects on chloroplasts (including lamellae and envelope membranes), mitochondria, cell walls, vacuoles, plasma membranes, single membrane-bound bodies, and ribosomal activity (Figs. 1-16). The ability of TMV to alter phenolic, enzymatic and oxidative metabolism is suggested by the marked effects on mitochondria (Figs. 3-6, 8). TMV particles appear to prevade all areas of the infected mesophyll leaf and root tissue, indicating that the particles are able to move freely from cell-to-cell, once the TMV particles move into the tissues (Figs. 1-16).

\section{Abstract}

Twelve-week-old Nicotiana tabacum L. cv. "Little Turkish" tobacco plants were infected with tobacco mosaic virus (TMV) and ultrastructural effects in leaf and root tissue were examined with respect to TMV effects on cellular organelles. TMV infected mesophyll tissue contain swollen chloroplasts with bulging out, electron dense opaque bodies and distorted lamellae. Mitochondria are enlarged with swollen cristae. The plasma membranes are quite electron dense and show discontinuities. TMV particles invade the cytoplasm of most TMV infected cells with evidence of heightened ribosomal activity. "Doughnut"-shaped nucleoli are present in infected cell nuclei with the chromatin becoming more granular in appearance. Fine structural examples of TMV infected leaf and root cells are discussed.

\section{References}

Betto, E., Bassi, M., Favali, M. A. and Conti, G. G. 1972. An electron microscopic and autoradiographic study of tobacco leaves infected with the U5 strain of tobacco mosaic virus. Phytopath. Z. 75: 193-201.

Boardman, N. K. and Zaitlin, M. 1958. The association of tobacco mosaic virus with plastids II. Studies on the biological significance of virus isolated from a chloroplast fraction. Virology 6: 758 .

Brants, D. H. 1964. The susceptibility of tobacco and bean leaves of tobacco mosaic virus infection in relation to the condition of ectodesmata. Virology 23: 588-594.

Cech, M. 1967. On the role of chloroplasts in the tobacco mosaic virus reproduction. Phytopathol. Z. 59: 72-82.

Conti, G. G., Bellini, E., Vegetti, G. and Favali, M. A. 1974. Changes in phenylalanine ammonia-

The chloroplast (C), bordering the large vacuole (VA), appears distorted and swollen with enlarged lammelae $(\mathrm{L})$ in disarray. Osmium tetroxide fixation with uranyl acetate post-staining. $\times 36,000$. 16, mesophyll leaf infected with TMV shows lesion that has formed outside of cell wall (CW). There are areas of vesiculation (VE) within the lesion where dissolution is occurring. The plasma membrane (PM) bordering the cell wall (CW) has become discontinuous (arrows). Osmium tetroxide fixation with uranyl acetate post-staining. $\times 58,000$. 
lyase and development of induced resistance in Nicotiana glutinosa leaves infected with the U5 strain of tobacco mosaic virus. Riv. Pat. Veg., SIV. 10: 177-193.

Dvorak, M. 1971. Submicroscopic cytodifferentiation. Erg. Anat. 45: 1-118.

Edwardson, J. R. 1966. Electron microscopy of cytoplasmic inclusions in cells infected with rodshaped viruses. Am. J. Bot. 53(4): 359-364.

Esau, K. 1933. Pathologic changes in the anatomy of leaves of the sugar beet, Beta valgaris L., affected by curly top. Phytopathology 23: 679-712.

- 1941. Phloem anatomy of tobacco affected with curly top and mosaic. Hilgardia 13: 437.

- and Cronshaw, J., 1967. Tubular components in cells of healthy and tobacco mosaic virusinfected Nicotiana. Virology 33: 26-35.

Farkas, G. L., Kiraly, Z. and Solymosy, F. 1960. Role of oxidative metabolism in the localization of plant viruses. Virology 12: 408-421.

Favali, M. A., Bass, M. and Conti, G. G. 1974. Morphological, cytochemical and autoradiographic studies of local lesions induced by the U5 strain of tobacco mosaic virus in Nicotiana glutinosa L. Riv. Pat. Veg., SIV. 19: 177-193.

Fritig. B., Legrand, M. and Hirth, L. 1972. Changes in the metabolism of phenolic compounds during the hypersensitive reaction of tobacco to TMV. Virology 47: 845-848.

Gerola, F. M., Bassi, M., Augusta, M. A. and Betto, E. 1969. An electron microscopy study of the penetration of tobacco mosaic virus into leaves following experimental inoculation. Virology 38: 380-386.

Goldstein, B. 1926. A cytological study of the leaves and growing points of healthy and mosaic diseased tobacco plants. Bull. Torrey. Botan. Club 53: 499.

Goodman, R. N., Kiraly, Z. and Zaitlin, M. 1967. The Biochemistry and Physiology of Infectious Plants Disease. D. van Nostrand Co., Princeton, NJ.

Henry, E. W., Valdovinos, J. G. and Jensen, T. E. 1971. Ethylene enhanced peroxidases in abscission cells of tobacco flower pedicels. Plant Physiol. 47: 83.

- and Jensen, T.E. 1973. Peroxidases in tobacco abscission zone tissue I. Fine structural localization during the ethylene-induced abscission. J. Cell Sci. 13: 591-601.

- 1974. Ultrastructural localization of polyphenoloxidase activity in chloroplasts of Nicotiana tabacum. Plant Physiol. 53 (Supplement): 63.

- , Valdovinos, J. G. and Jensen, T. E. 1974. Peroxidases in tobacco abscission zone tissue II. Time course studies of peroxidase activity during ethylene-induced abscission. Plant Physiol. 54: 192-196.

- 1975a. Polyphenol oxidase activity in thylakoids and membrane-bound granular components of Nicotiana tabacum chloroplasts. J. de Microscopie 22(1): 109-116.

- 1975b. Peroxidases in tobacco abscission zone tissue III. Ultrastructural localization in the thylakoids and membrane-bound bodies of chloroplasts. J. Ultrastruct. Res. 52: 289-29

- 1978. An ultrastructdral study of ethylene-treated stem segments of Alaska pea (Pisum sativum). Cytologia 43: 423-432.

Holmes, F. O. 1929. Local lesions in tobacco mosaic. Contributions of the Boyce Thompson Institute. 1: 504-520.

Israel, H. W. and Ross, A. F. 1967. The fine structure of local lesions induced by tobacco mosaic virus in tobacco. Virology 33: 272-286.

Jensen, T. E. and Valdovinos, J. G. 1967. Fine structure of abscission zones I. Abscission zones of the pedicles of tobacco and tomato flowers at anthesis. Planta (Berl.) 83: 303-313.

Kolehmainen, I., Zech, H. and Von Wettstein, D. 1965. The strcutre of cells during tobacco mosaic virus reproduction. Mesophyll cells containing virus crystals. J. Cell Biol. 25: 77.

Kontaxis, D. G. and Schlegel, D. E. 1962. Basal septa of broken trichomes in Nicotiana as possible infection sites for tobacco mosaic virus. Virology 16: 244-247.

Luft, J. H. 1961. Improvements in epoxy resin embedding methods. J. Biophys. Biochem. Cytol. 9: $409-414$.

Milne, R. G. 1966. Multiplication of tobacco mosaic virus in tobacco leaf palisade cells. Virology 28: 79-89. 
Reddi, K. K. 1964. Studies on the formation of tobacco mosaic virus ribonucleic acid V. Presence of tobacco mosaic virus in the nucleus of the host cell. Proc. Natl. Acad. Sci. U. S. 52: 397.

Shalla, T. A 1968. Virus particles in chloroplasts of plants infected with the U5 strain of tobacco mosaic virus. Virology 35: 194-203.

Weintraub, M. and Ragetli, H. W. J. 1964. An electron microscope study of tobacco mosaic virus lesions in Nicotiana glutinosa L. J. Cell Biol. 23: 499-509.

Weststeijn, E. A. 1976. Peroxidase activity in leaves of Nicotiana tabacum var. Xanthi nc. before and after infection with tobacco mosaic virus. Physiol. Plant. Pathol. 8: 63-71.

Wu, J. H. 1973. Wound-healing as a factor in limiting the size of lesions in Nicotiana glutinosa leaves infected by the very mild strain of tobacco mosaic virus (TMV-VM). Virology 51: $474-484$.

Zaitlin, M. and Boardman, N. K. 1958. The association of tobacco mosaic virus with plastids I. Isolation of virus from chloroplast fraction of diseased-leaf homogenates. Virology 6 : 743. 